\title{
Introduction for Volume 10, Issue 2
}

This issue contains 6 papers. There are 4 contributions in English and 2 contributions in Chinese with English abstracts. The papers can be divided into four topics: COVID-19, disaster psychological crisis, risk analysis and risk assessment

In this issue, two papers are in COVID-19 Topic. The first paper "Analysis of Death Risk of COVID-19 under Incomplete Information" by Chongfu Huang, suggest a hybrid model to analyze the death risk under incomplete information. The hybrid model would be supported by the internet of intelligences, being a platform interacting with infectious disease specialists and local doctors who fuse the evidences with the experience of the known infectious diseases and provide a series of judgments related to the death risk of a human population in a given period to COVID-19. The paper gives a virtual case to show how to use the hybrid model. The second paper "Research on the prevention and control of COVID-19 risk in Long-term care facilities based on Prospect Theory " by Fengying Zhang, et al., attempts to explore the uncertain relationship between the identification of the risk of COVID-19 and the management and care behavior of the elderly care institutions, aiming to achieve management optimization.

There is one paper in disaster psychological crisis. The paper “Mutation Mechanism of Victim's Psychological Behavior State After Major Natural Disasters" by Xuanhua Xu, et al., analyzed the victim's psychological and behavioral characteristics and influencing factors during recovery stage based on lots of literatures and an interview with victims. Besides, a mechanism chart of the formation of the victim's psychological behavior and a cusp catastrophe model are constructed. The model is partially verified by the investigation data of the disaster area. Finally, the paper put forward some strategies to cope with victim's psychology and behavior during recovery stage based on the cusp catastrophe model analysis.

There are two papers in risk analysis. The first paper "Patterns of Fatal Explosion Accidents in China" by Siheng Sun, et al., explores the patterns of fatal explosion accidents (FEA). Statistical analysis was made based on the year, month, time interval of day, day of week, province, type of accident, place of occurrence and severity of accident by using the database containing 1,835 FEA that happened in China during 2001-2018. The second paper “An analysis method of black swan event based on impact-spread tree”by Xinying Liu \& Hang LI, solved the problem of whether a method can be used to quantitatively determine whether an event can be called a black swan event before it is defined as a black swan event. This paper proposes a set of analysis methods to determine the black swan event by constructing an impact-spread tree. The case study shows that the method of impact-spread tree can effectively distinguish between black swan events and non-black swan events.

There is one paper in risk assessment, "A Sales Forecasting Model for the Consumer Goods with Holiday Effects" by Mu Zhang, et al., which proposes a sales forecasting model for the consumer goods with holiday effects by introducing the seasonal decomposition and ARIMA model. Firstly, a dummy variable model is constructed to test the holiday effects in consumer goods market. Secondly, using the seasonal decomposition, the seasonal factor is separated from the original series, and the seasonally adjusted series is then obtained. Through the ARIMA model, a trend forecast to the seasonally adjusted series is further carried out. Finally, according to the multiplicative model, refilling the trend forecast value with the seasonal factor, thus, the final sales forecast results of the consumer goods with holiday effects can be obtained.

We sincerely thank the referees for their strong support and kind help. Thanks to all the authors for their submissions. Particularly, thanks to Prof. Mu Zhang, being technology editor, and thanks to Prof. Junxiang Zhang, Manager of Journal of Risk Analysis and Crisis Response, they devoted their time to overseeing the reviews.

Editors-in-Chief:

\author{
Prof. Chongfu Huang \\ Beijing Normal University, No.19 Xinjiekouwai Street, Beijing 100875, China \\ Email:hchongfu@126.com
}

\section{Prof. Gordon Huang}

Faculty of Engineering and Applied Science, University of Regina,

Regina, Sask S4S 0A2, Canada

Email:gordon.huang@uregina.ca 